\title{
Thermoplastic sugar palm starch composites
}

\begin{abstract}
Chapter 9 discusses the development of biodegradable materials derived from sugar palm starch. Due to the increasing environmental problems arising from the disposal of nonbiodegradable plastics, the development of biopolymers has gained increasing attention from researchers as substitutes for conventional petroleum-based plastic. Among biopolymers, starch has been considered the most promising material because it is low in cost, highly abundant, renewable and fully biodegradable. This biopolymer can be transformed into thermoplastic starch in the presence of heat and plasticizer. Recent research has focused on the development of thermoplastic starch utilizing a new source of starch, sugar palm (Arenga pinnata). Unfortunately, thermoplastic sugar palm starch presents some drawbacks, such as poor mechanical properties, high sensitivity to moisture, and low thermal degradation. Therefore, various studies have been carried out to overcome these drawbacks, such as blending thermoplastic sugar palm starch with other biopolymers and reinforcement with various natural fibers to enhance the properties of this bio-based material.
\end{abstract}

